\title{
A Retrospective Analysis of the Efficacy and Safety of Anlotinib in the Treatment of Advanced Sarcoma
}

Qiang Yan ( $\nabla$ yq09100319@163.com )

Affiliated Tumor Hospital of Zhengzhou University: Henan Cancer Hospital https://orcid.org/00000001-6102-7053

Xinhui Du

Affiliated Tumor Hospital of Zhengzhou University: Henan Cancer Hospital

Liangyu Guo

Affiliated Tumor Hospital of Zhengzhou University: Henan Cancer Hospital

Weitao Yao

Affiliated Tumor Hospital of Zhengzhou University: Henan Cancer Hospital

\section{Research Article}

Keywords: Anlotinib, osteosarcoma, soft tissue sarcoma, efficacy, safety

Posted Date: May 19th, 2021

DOI: https://doi.org/10.21203/rs.3.rs-506500/v1

License: (1) (1) This work is licensed under a Creative Commons Attribution 4.0 International License.

Read Full License 


\section{Abstract}

Background: Patients with advanced sarcomas have a dismal prognosis with few effective therapies. Previous research has demonstrated the efficacy of anlotinib in the treatment of advanced sarcomas. However, there are few relevant clinical studies, and the efficacy of anlotinib varies among sarcomas of different subtypes. Therefore, more clinical studies are needed to explore the efficacy of anlotinib in different subtypes of sarcomas. This study assessed the efficacy and safety of anlotinib monotherapy in the treatment of advanced sarcoma.

Methods: Data from 45 advanced sarcoma patients who received anlotinib monotherapy at Affiliated Cancer Hospital of Zhengzhou University between June 2018 and February 2021 were retrospectively analyzed, including 5 cases of osteosarcoma and 40 cases of soft tissue sarcoma(STS). According to Response Evaluation Criteria In Solid Tumors (RECIST) Version 1.1, we evaluated objective response rates (ORR) and disease control rates (DCR) at 3 months, as well as overall ORR and DCR, and calculated progression-free survival(PFS), and then evaluated treatment-related adverse events (AEs).

Results: 44 patients were evaluated for efficacy and 45 for treatment-related AES. The ORR and DCR after 3 months were $6.82 \%$ and $81.82 \%$ respectively. At the end of follow-up, the overall ORR was $2.27 \%$, the total DCR was $27.27 \%$, and the median progression-free survival (m-PFS) was 5.71 months. Among them, the m-PFS of alveolar soft tissue sarcoma (ASPS) was 8.07 months, which was significantly longer than that of other subtypes of sarcoma $(P=0.025)$. The most common adverse events were hypothyroidism (increased TSH) (17.8\%), anemia (15.6\%), fatigue (11.1\%), loss of appetite (11.1\%), decreased liver function (11.1\%), leukopenia (8.9\%) and hand-foot syndrome (8.9\%). After treatment, 5 patients developed grade $3 \mathrm{AES}$, including decreased liver function (4.4\%), hypertension (2.2\%), proteinuria (2.2\%), fatigue (2.2\%), and loss of appetite (2.2\%). One patient had severe myelosuppression and a significant decrease in white blood cells and platelets. It is worth noting that the PFS of patients with hand-foot syndrome after treatment was significantly longer than that of patients without hand-foot syndrome $(\mathrm{P}<$ 0.05).

Conclusion: Anlotinib is effective in the treatment of sarcoma, especially in ASPS, Synovial sarcoma (SS) and Fibrosarcoma (FS), and its toxicity is controllable. In addition, the occurrence of hand-foot syndrome after treatment may be related to a good prognosis. However, large sample and prospective studies are needed to confirm it.

\section{Introduction}

Sarcoma is a malignant tumor originating from mesenchymal tissue. More than 70 histological subtypes have been reported[1, 2], including osteosarcoma and soft tissue sarcoma (STS). The incidence of sarcoma is low, accounting for about $1 \%$ of malignant tumors in adults and $15 \%$ of malignant tumors in children [3]. However, in 2014, there were more than 39,900 new cases of sarcoma in China, accounting 
for $1.05 \%$ of the total incidence of cancer [4]. The common primary sites of sarcoma are extremities, trunk, retroperitoneum and so on.

For early, locally resectable sarcomas, surgery is the main treatment [5]. However, nearly $50 \%$ of patients have local recurrence and distant metastasis after surgery, and some patients have metastases at the first visit, and the common sites of metastasis are lung, liver and brain [6, 7]. For patients with advanced metastatic or unresectable sarcomas, systemic chemotherapy is the most commonly used method [8]. The common chemotherapeutic drugs for advanced osteosarcoma include adriamycin, methotrexate, cisplatin, and ifosfamide, etc., and most chemotherapy regimens for advanced STS are doxorubicin alone or adriamycin combined with ifosfamide $[9,10]$. However, the prognosis of patients with stage IV sarcoma is extremely poor, the median overall survival ( $\mathrm{m}-\mathrm{OS}$ ) is about 12 months, and the 5-year survival rate is less than $10 \%$ [10-12]. In addition, the effective rate of chemotherapy for advanced sarcoma is only $14-48 \%$, and long-term use of cytotoxic drugs such as adriamycin will increase the risk of cardiomyopathy $[13,14]$. Therefore, it is necessary to find treatments that can improve survival and safety.

Pathological angiogenesis plays an important role in the growth, progression and metastasis of sarcoma, so anti-angiogenesis is very important to control the progression of sarcoma[12, 15]. Previous studies have shown that vascular endothelial growth factor (VEGF) and its receptor (VEGFR) are involved in angiogenesis in sarcomas. Tyrosine kinase inhibitors (TKIs) targeting VEGFR have been developed clinically and have shown anti-tumor activity in a variety of malignant tumors including sarcomas[16, 17]. Since pazopanib was approved for STS by the Food and Drug Administration (FDA) in 2012[18], many TKIs have been used in the clinical treatment of sarcoma, including pazopanib [19], regorafenib [20, 21], apatinib [17, 22], etc.

Anlotinib is a novel type of multi-target tyrosine kinase inhibitor, which has the effect of anti-tumor proliferation and anti-angiogenesis. Anlotinib has a strong inhibitory effect on VEGFR-2 and-3, in addition, Anlotinib has a strong inhibitory effect on fibroblast growth factor receptors (FGFR)-1,-2,-3 and FGFR4. It has been reported that anlotinib shows good anti-tumor activity against advanced non-small cell lung cancer, small cell lung cancer, renal clear cell carcinoma, medullary thyroid carcinoma and other tumors [23-26]. In a phase II clinical trial [27], 166 patients with advanced sarcoma were treated with anlotinib and showed significant efficacy. In this trial, the 12-week progression-free rate (PFR) was $68 \%$, the ORR was $13 \%$, the m-PFS was 5.6 months, and the m-OS was 12 months. Compared with placebo, anlotinib prolonged PFS and improved the ORR and DCR. Tian et al. reported that the ORR, DCR and m-PFS were $0 \%, 23 \%$ and 2.7 months in patients with osteosarcoma after treatment with anlotinib, while the ORR, DCR and $\mathrm{m}$-PFS of STS patients were $14 \%, 55 \%$ and 6 months [13].

These two studies suggest that anlotinib is a good treatment option for patients with advanced metastatic sarcoma. However, there are some problems in the above two studies. First of all, there are many subtypes of sarcoma, and fewer patients are included in each subtype, which makes it impossible to accurately determine the sensitivity of different subtypes of sarcoma to anlotinib. Secondly, there are 
different tumorigenesis and progression mechanisms between osteosarcoma and STS, so it is necessary to analyze the difference of curative effect between the two groups. Third, there are few reports about the efficacy and safety of anlotinib in the treatment of advanced sarcoma, and the efficacy of different studies are different. Therefore, more studies are needed to clarify the efficacy and safety of anlotinib, and explore the efficacy differences between different subtypes of sarcoma. The purpose of this study was to explore the efficacy and safety of anlotinib monotherapy in the treatment of advanced metastatic sarcoma, and to analyze the difference in efficacy among patients with different subtypes of sarcoma, so as to provide more comprehensive treatment options for sarcoma patients.

\section{Patients And Methods \\ Patients and eligibility criteria}

This present study is a single-center retrospective study to evaluate the efficacy and safety of anlotinib monotherapy for advanced metastatic sarcoma patients. From June 2018 to February 2021, 45 patients with advanced sarcoma who had failed in prior treatment were treated with anlotinib monotherapy at the Affiliated Cancer Hospital of Zhengzhou University. The patient eligibility criteria included the following: 1) histologically proven osteosarcoma or STS; 2 ) initial treatment in the orthopedic oncology department of the Affiliated Cancer Hospital of Zhengzhou University; 3) no previous malignancy; 4) unresectable local advanced lesions or multiple metastatic lesions that could not be cured by local therapy; 5) with good Eastern Cooperative Oncology Group Performance Status $(\leq 2) ; 6)$ measurable lesions according to RECIST1.1; 7) It can accept the examination of hematologic, hepatic, and renal function. 8) The clinical data are complete and can be analyzed statistically. This study was performed in accordance with the Declaration of Helsinki, and it was approved by the Ethics Committee of Cancer Hospital Affiliated to Zhengzhou University.

\section{Treatment methods}

All patients with advanced sarcoma who failed prior treatment were treated with anlotinib monotherapy. Anlotinib was initially administered orally at a dosage of $12 \mathrm{mg}$ or $10 \mathrm{mg}$ daily for 14 days and then discontinued for 7 days (3-week cycle). The specific initial drug dose was determined by the clinicians depending on the patient's condition and tolerance. Dose reduction $(10 \mathrm{mg} / 8 \mathrm{mg})$ was allowed for drugrelated grade 3/4 AEs. Treatment continued until disease progression or occurrence of unacceptable toxicities (grade 3/4 AEs) or death.

\section{Efficacy and safety evaluation}

Follow up plan, tumor response, drug efficacy, survival time and AES were evaluated and recorded. The target lesions were evaluated with computed tomography (CT) or magnetic resonance imaging (MRI) every 2-3 months, but immediately if clear signs of disease progression (PD) were observed. According to RECIST version 1.1, tumor response was divided into complete response (CR), partial response (PR), disease stability (SD) and PD. Then PFS, ORR and DCR were evaluated. PFS was the primary endpoint, 
and ORR and DCR at 3 months were serving as secondary endpoints. PFS was defined as the interval from onset of anlotinib administration to disease progression or death from any cause. The ORR was calculated as $(C R+P R) /$ total number of cases. The $D C R$ was calculated as $(C R+P R+S D) /$ total number of cases.

Safety and toxicity assessments were performed in all patients treated with anlotinib. Treatment-related AEs were assessed and graded according to the National Cancer Institute Common Terminology Criteria for Adverse Events (version 4.03).

\section{Statistical analysis:}

The classified variables were expressed as number and percentage. Normally distributed quantitative data were shown as mean and standard deviation, while non-distributed quantitative data were expressed as median and range. PFS were calculated by using the Kaplan-Meier method, with the log-rank test used for subgroup comparison. All statistical analyses were two-sided, and significance was set at $P<0.05$. All data were analyzed with the SPSS 26.0 statistical package.

\section{Results}

\section{Patient information:}

From June 2018 to February 2021, 45 patients with advanced sarcoma were treated with anlotinib monotherapy at the Affiliated Cancer Hospital of Zhengzhou University. The basic clinical characteristics of the patients are shown in Table 1. The median age of all patients was 43 years (8-79 years), including 29 male patients (64.4\%) and 16 female patients (35.6\%). Pathological subtypes included synovial sarcoma (SS) in 8 cases (17.8\%), alveolar soft tissue sarcoma (ASPS) in 7 cases (15.6\%), osteosarcoma in 5 cases (11.1\%), fibrosarcoma (FS) in 5 cases (11.1\%), undifferentiated pleomorphic sarcoma (UPS) in 4 cases $(8.9 \%)$, unknown type sarcoma in 4 cases (8.9\%), rhabdomyosarcoma (RS) in 2 cases $(4.4 \%)$ and leiomyosarcoma (LS) in 2 cases (4.4\%), There were chordoma, epithelioid sarcoma (ES), clear cell sarcoma, small round cell sarcoma, Ewing sarcoma and liposarcoma in 1 case (2.2\%). The primary tumors were located in the extremities in 25 cases (55.6\%) and trunk in 20 cases (44.4\%). Table 2 shows the prior treatment information of all patients. It can be seen that the majority of patients $(36 / 45,80.0 \%)$ received surgical resection, 25 patients (55.6\%) received chemotherapy, while 41 patients $(93.2 \%)$ developed lung metastasis. In addition, statistics on the sensitivity of patients to chemotherapy showed that most patients $(23 / 45,51.1 \%)$ were normally sensitive to chemotherapy, only 3 patients $(3 / 45,6.7 \%)$ were very sensitive to chemotherapy, while 12 patients $(12 / 45,26.7 \%)$ were not sensitive to chemotherapy. According to the statistics of lines of therapy for the treatment of sarcoma with anlotinib, 3 patients $(3 / 45,6.7 \%)$ received the first-line treatment with anlotinib, 13 patients $(13 / 45,38.9 \%)$ received the second-line treatment, and 29 patients $(29 / 45,64.4 \%)$ received the third-line treatment or more. Most of the patients (80.0\%) were in good condition, and the Eastern Cooperative Oncology Group (ECOG) score was 0,1 and 2 . 


\section{Effectiveness:}

\section{General results}

Of the 45 patients treated with anlotinib, 44 received at least one complete cycle of treatment and were included in the efficacy evaluation. One patient with undetermined subtype of sarcoma was treated for less than one cycle, so only safety and adverse reactions were evaluated. By February 2021, the follow-up period ranged from 0.90 to 18.10 months, with an average follow-up time of 8.10 months.

\section{Short-term efficacy}

The changes of target lesions in all patients are shown in Fig. 1. After 3 months of treatment with anlotinib, no CR was obtained. Three patients were evaluated as PR, including 2 synovial sarcomas and 1 patient with no specific sarcoma subtype. 33 patients were evaluated as SD, and only 8 patients were assessed as PD, with an ORR of $6.82 \%$ and a DCR of $81.82 \%$ (as shown in Table 3 ). Therefore, $81.82 \%$ of the patients showed a certain response to anlotinib monotherapy.

\section{Long-term efficacy}

44 patients received the final efficacy evaluation (Fig. 1, table 3). According to RECIST version 1.1, there was no CR in 44 patients, 1 patient was evaluated as PR, 11 patients as SD, and 32 patients as PD. The total ORR was $2.27 \%$, and the total DCR was $27.27 \%$. The m-PFS was 5.71 months (Fig. 2a), and the mOS was not obtained.

The factors affecting PFS were analyzed as follows: a. There was no statistically significant difference in PFS based on the patient's history of targeted therapy and the dose of anlotinib $(P<0.05)(F i g .2 b-2 c) ; b$. There were significant differences in PFS among different clinical results. PFS was significantly prolonged in PR group $(P=0.00)$ (Fig. 2e); c. Different histological types: The m-PFS of ASPS group was the longest, reaching 8.07 months. The m-PFS of SS, FS, UPS and osteosarcoma groups were 7.33, 5.15, 3.32 and 2.86 months, respectively. There were significant differences among different subtypes $(P=$ 0.025) (Fig. 2f).

Fig. 3 shows the changes in pulmonary metastasis in a 46-year-old male patient with SS before and after treatment. The patient was diagnosed with right leg SS with lung metastasis and had progressive disease after surgeries and standard Adriamycin + Ifosfamide (Al) chemotherapy. From September 2019 to November 2020, he was administered with anlotinib. (a) pulmonary lesion of the patient before treatment; (b) pulmonary metastasis at months 14 after the treatment. It is clear that the pulmonary metastasis after the drug treatment has been significantly reduced and accompanied by cavity formation.

\section{Safety and toxicity}

The safety of 45 patients treated with anlotinib was evaluated, and 29 patients (64.4\%) had adverse events (Table 4, Fig. 4). The majority of patients had mild adverse events, but a few patients had grade 
$3 / 4$ adverse events. Overall, grade 1 (60.8\%) and grade 2 (23.5\%) adverse events accounted for $84.3 \%$ of all AES, while grade $3(11.8 \%)$ and grade $4(3.9 \%)$ accounted for $15.7 \%$ of all AES. The common adverse events were hypothyroidism (increased TSH) $(8,17.8 \%)$, anemia $(7,15.6 \%)$, fatigue $(5,11.1 \%)$, loss of appetite $(5,11.1 \%)$, decreased liver function $(5,11.1 \%)$, leukopenia $(4,8.9 \%)$ and hand-foot syndrome $(4$, $8.9 \%)$.

However, 5 patients (11.1\%) had grade 3 adverse events, and 1 patient had grade 3 hypertension and fatigue at the same time. The common grade 3 adverse reactions were decreased liver function $(2,4.4 \%)$, hypertension $(1,2.2 \%)$, proteinuria $(1,2.2 \%)$, fatigue $(1,2.2 \%)$ and loss of appetite $(1,2.2 \%)$. For these 5 patients with grade 3 adverse events, the treatment of anlotinib was not stopped. After dose reduction and supportive symptomatic treatment, the adverse reactions gradually improved. Therefore, it is necessary to monitor the drug toxicity and adjust the drug dose in time. Notably, one patient developed severe myelosuppression with significantly decreased white blood cells and platelets, and was assessed to have grade 4 AES. The patient was forced to discontinue treatment and received symptomatic antimyelosuppression treatment. No patient died of AES.

The relationship between adverse events and effectiveness was evaluated, and it was found that the PFS of 4 patients with hand-foot syndrome was significantly longer than that of the patients without handfoot syndrome (12.55 months vs 5.07 months, $P=0.036)$ (Fig. 2d).

\section{Discussion}

Sarcoma is a rare malignant tumor originated from mesenchymal tissue. For advanced, unresectable sarcomas, systemic chemotherapy is the most common method. However, the m-OS of patients with advanced sarcoma is about 12 months, and the 5-year survival rate is less than 10\% [10]. In addition, the sensitivity of different subtypes of sarcoma to chemotherapy is different, and the adverse events of chemotherapy are large, which hinders the application of systemic chemotherapy. Therefore, it is urgent to find a more effective treatment for patients with advanced sarcoma.

A large number of studies have confirmed that angiogenesis plays a significant role in the occurrence, progression and metastasis of tumor [15]. It has been reported that overexpression of VEGF is associated with early recurrence, metastasis and low survival rate of sarcoma [28]. Therefore, targeted therapy for VEGF / VEGFR is a feasible treatment. At present, small molecule targeted drugs TKIs have been used in the treatment of sarcoma, and have shown good results, including pazopanib, apatinib, sunitinib, and anlotinib [20, 27, 29-32]. Pazopanib is the only antiangiogenic drug approved for sarcoma. In the palette clinical trial of pazopanib [33], $6 \%$ of patients were evaluated as PR after pazopanib was used, $67 \%$ were evaluated as SD, and $23 \%$ were evaluated as PD, with m-PFS of 4.6 months. Another pazopanib clinical study [30] reported that $15.6 \%$ of patients were evaluated as PR, $39.2 \%$ were assessed as SD, m-PFS and m-OS were 5.3 months and 12 months respectively. Two other studies on pazopanib have also produced similar results [34, 35]. Liu et al. [22] reported that the ORR, m-PFS and m-OS of apatinib in the treatment of sarcoma were $23.68 \%, 7.87$ and 17.55 months, respectively. Several other studies of apatinib also 
reported similar results $[13,17,36,37]$. There are also studies reported that after treatment with sunitinib, $1.8 \%$ of patients reached PR, and $20 \%$ of patients achieved SD [38].

Anlotinib is a novel oral drug targeting VEGFR-1/-2/-3, platelet-derived growth factor receptor $a / \beta, c-k i t$ and other targets. It has dual effects of inhibiting tumor cell proliferation and tumor angiogenesis.It has been approved by China Food and Drug Administration for second-line treatment of advanced and metastatic sarcoma in 2019 [39, 40]. Chi et al. [27] reported that anlotinib showed a good therapeutic effect in the treatment of sarcoma. A total of 166 patients were enrolled in the study, with ORR of $13 \%, \mathrm{~m}$ PFS of 5.6 months, and m-OS of 12 months. Patients with different subtypes of sarcoma showed different therapeutic effects.

This study evaluated the efficacy and safety of monotherapy with anlotinib in 45 patients with sarcoma. The results showed that the ORR and DCR were $6.82 \%$ and $81.82 \%$ after 3 months of treatment. The total ORR, DCR and m-PFS were $2.27 \%, 27.27 \%$ and 5.71 months after the end of treatment. In this study, the efficacy of anlotinib was slightly better than pazopanib and sunitinib, but similar to that of apatinib in the treatment of sarcoma, DCR and m-PFS were similar. Unfortunately, the ORR in this study is lower $[13,17$, $22,30,33-38]$. In addition, the efficacy of this study is similar to that reported by Chi [27] in the treatment of sarcoma. Although ORR is low, m-PFS is slightly longer.

In this study, the analysis of the influencing factors of PFS showed that there were differences in the efficacy of different subtypes of sarcomas treated with anlotinib. The efficacy of ASPS patients was the best, and the m-PFS reached 8.07 months, which was significantly better than that of other subtypes of sarcomas. Previous studies have also reported the good efficacy of TKI in the treatment of ASPS. For example, Stacchiotti $S$ et al.[41] reported that the m-PFS of sunitinib in the treatment of ASPS was 17 months. Also, Stacchiotti S et al. [42] reported that the m-PFS of pazopanib in the treatment of ASPS was 13.6 months, and Wang et al reported that the m-PFS reached 18.53 months after apatinib in the treatment of ASPS, while in another study of anlotinib in the treatment of ASPS, m-PFS even reached 21 months [27]. The reason why the therapeutic effect of ASPS is better than that of other subtypes of sarcoma after TKI treatment is not completely clear, and it is believed that it may be related to the following reasons: First, ASPS is considered to be an inert sarcoma [43], so the survival time of patients with ASPS is longer than that of patients with other subtypes of sarcoma; Secondly, due to the high heterogeneity of sarcomas, the sensitivity of different subtypes of sarcomas to TKI is different, so it is necessary to further study the sensitivity of different sarcoma targets.

A number of studies have reported that the ORR of metastatic STS treated with apatinib is higher than that of osteosarcoma, while there is no significant difference in DCR, m-PFS, m-OS between the two groups [12, 22, 44]. However, Zhu et al. [36] reported that compared with osteosarcoma, apatinib has a tendency to improve the PFS of STS. In this study, the m-PFS of osteosarcoma after the administration of anlotinib was 2.86 months, and the curative effect was not as good as that of ASPS, SS, FS and UPS, which was similar to what Zhu et al. reported. The reason for the difference between the efficacy of anlotinib in the treatment of osteosarcoma and STS is not clear, which may be related to the following 
reasons: Firstly, the biological characteristics of osteosarcoma and STS are different, which leads to different sensitivity to anlotinib [29, 45]; Secondly, the prevalence of osteosarcoma is different from that of STS. Osteosarcoma is more common in children and adolescents, while STS is more common in adults. Due to the differences in physical condition, physiological mechanism and immune function between children and adults, it may lead to different therapeutic effects after the application of anlotinib. Thirdly, there are also obvious differences in genetics and genomics, which may lead to differences in sensitivity and efficacy of targeted drugs [46-48].

In this study, the safety of anlotinib monotherapy in the treatment of sarcoma is good, most patients only have grade 1 and 2 adverse events. The most common adverse events were hypothyroidism (increased TSH), anemia, fatigue, loss of appetite, decreased liver function, leukopenia and hand-foot syndrome, which were similar to the previously reported adverse reactions of anlotinib in the treatment of sarcoma and lung cancer $[11,27,49,50]$. It is worth noting that one patient developed severe myelosuppression, which was also reported in the study of Wang et al. [11]. Therefore, during the treatment, it is necessary to timely monitor the blood routine and other examinations of the patients, and timely find and deal with myelosuppression.

Liu et al. [22] found that the OS of patients with AES such as hypertension, hand-foot syndrome and proteinuria after treatment with apatinib was longer than that of patients without these AES, while there was no significant difference in ORR, DCR and PFS. Liao et al. [12] also reported similar findings. In this study, many patients did not achieve OS, so the relationship between AES and OS could not be evaluated. However, in this study, the PFS of 4 patients with hand-foot syndrome was significantly longer than that of the patients without hand-foot syndrome (12.55 months vs 5.07 months, $P=0.036$ ) (Fig. 2D). The reasons for the relationship between AES and clinical outcomes are still unclear, and further studies are needed to clarify the relationship.

There are some limitations in this study. First of all, this study is a retrospective study, and there is no control. Secondly, there are few patients in some subtypes. Because of the heterogeneity of sarcomas, the results may be affected in the efficacy analysis among different subtypes. Thirdly, the patients received different treatment regimens, which may have an impact on the results of this study. Finally, this study was limited to clinical evaluation, and no target and molecular biology monitoring studies were conducted.

\section{Conclusion}

The therapeutic effect of anlotinib is considerable, especially in ASPS, SS and FS, and its toxicity is controllable. In addition, the emergence of hand-foot syndrome may be related to clinical efficacy. But large sample and prospective studies are needed to further confirm the anti-tumor activity of anlotinib, so as to benefit more patients with sarcoma.

\section{Declarations}


Funding This research did not receive any specific grant from funding agencies in the public, commercial, or not-for-profit sectors.

Conflicts of interest/Competing interests The authors declare that they have no competing interests.

Availability of data and materials All data generated or analysed during this study are included in this published article.

Code availability Not applicable

Authors' contributions All authors participated in the study. Weitao Yao and Xinhui Du contributed to the conception and design of the research, Qiang Yan and Liangyu Guo contributed to the acquisition of the data, Qiang Yan and Weitao Yao contributed to the analysis and interpretation of the data. Qiang Yan contributed to the drafting of the manuscript. Qiang Yan, Xinhui Du and Weitao Yao contributed to the revision of the manuscript. All authors read and approved the final manuscript.

Ethics approval The study was conducted in accordance with the principles of the Declaration of Helsinki, and the study protocol was approved by the ethics committee of affiliated cancer hospital of zhengzhou university.

consent to participate Formal consent was not required for this type of study.

Consent for publication Not applicable

Compliance with ethical standards

Disclosure of potential conflicts of interest All authors who took part in this study indicate that they do not have anything to declare regarding funding or conflicts of interest with respect to this study.

Research involving Human Participants and/or Animals Not applicable

Informed consent Individually informed consent was waived for this retrospective study.

Acknowledgements We are grateful to all the patients and investigators in this study. This research did not receive any specific grant from funding agencies in the public, commercial, or not-for-profit sectors.

\section{References}

1. Biermann JS, Chow W, Reed DR, et al (2017) NCCN Guidelines Insights: Bone Cancer, Version 2.2017. J Natl Compr Canc Netw 15(2): 155-167

2. Von Mehren M, Kane JM, Bui MM, et al (2020) NCCN Guidelines Insights: Soft Tissue Sarcoma, Version 1.2021. J Natl Compr Canc Netw 18(12): 1604-1612

3. Siegel RL, Miller KD, Jemal A (2018) Cancer statistics, 2018. CA Cancer J Clin 68(1): 7-30 
4. Yang Z, Zheng R, Zhang S, Zeng H, Li H, Chen W (2019) Incidence, distribution of histological subtypes and primary sites of soft tissue sarcoma in China. Cancer Biol Med 16(3): 565-574

5. Von Mehren M, Randall RL, Benjamin RS, et al (2018) Soft Tissue Sarcoma, Version 2.2018, NCCN Clinical Practice Guidelines in Oncology. J Natl Compr Canc Netw 16(5): 536-563

6. Singhi EK, Moore DC, Muslimani A (2018) Metastatic Soft Tissue Sarcomas: A Review Of Treatment and New Pharmacotherapies. P T 43(7): 410-429

7. Grimme F, Seesing M, van Hillegersberg R, et al (2019) Liver Resection for Hepatic Metastases from Soft Tissue Sarcoma: A Nationwide Study. Dig Surg 36(6): 479-486

8. Ratan R, Patel SR (2016) Chemotherapy for soft tissue sarcoma. Cancer 122(19): 2952-60

9. Liebner DA (2015) The indications and efficacy of conventional chemotherapy in primary and recurrent sarcoma. J Surg Oncol 111(5): 622-31

10. Tap WD, Papai Z, Van Tine BA, et al (2017) Doxorubicin plus evofosfamide versus doxorubicin alone in locally advanced, unresectable or metastatic soft-tissue sarcoma (TH CR-406/SARC021): an international, multicentre, open-label, randomised phase 3 trial. Lancet Oncol. 18(8): 1089-1103

11. Wang HY, Chu JF, Zhang P, et al (2020) Safety and Efficacy of Chemotherapy Combined with Anlotinib Plus Anlotinib Maintenance in Chinese Patients with Advanced/Metastatic Soft Tissue Sarcoma. Onco Targets Ther 13: 1561-1568

12. Liao Z, Li F, Zhang C, et al (2019) Phase II trial of VEGFR2 inhibitor apatinib for metastatic sarcoma: focus on efficacy and safety. Exp Mol Med 51(3): 1-11

13. Tian Z, Liu H, Zhang F, et al (2020) Retrospective review of the activity and safety of apatinib and anlotinib in patients with advanced osteosarcoma and soft tissue sarcoma. Invest New Drugs 38(5): 1559-1569

14. Ghignatti P, Nogueira LJ, Lehnen AM, Leguisamo NM (2021) Cardioprotective effects of exercise training on doxorubicin-induced cardiomyopathy: a systematic review with meta-analysis of preclinical studies. Sci Rep 11(1): 6330

15. Versleijen-Jonkers YM, Vlenterie M, van de Luijtgaarden AC, van der Graaf WT (2014) Anti-angiogenic therapy, a new player in the field of sarcoma treatment. Crit Rev Oncol Hematol 91(2): 172-85

16. English WR, Lunt SJ, Fisher M, et al (2017) Differential Expression of VEGFA Isoforms Regulates Metastasis and Response to Anti-VEGFA Therapy in Sarcoma. Cancer Res 77(10): 2633-2646

17. Liao Z, Li T, Zhang C, et al (2020) Clinical study of apatinib in the treatment of stage IV osteogenic sarcoma after failure of chemotherapy. Cancer Biol Med 17(2): 501-512

18. Nakano K, Takahashi S (2018) Current Molecular Targeted Therapies for Bone and Soft Tissue Sarcomas. Int J Mol Sci 19(3)

19. Koca S, Beşiroğlu M, Özçelik M, et al (2021) Pazopanib for metastatic soft-tissue sarcoma: A multicenter retrospective study. J Oncol Pharm Pract 27(3): 541-546

20. Mir O, Brodowicz T, Italiano A, et al (2016) Safety and efficacy of regorafenib in patients with advanced soft tissue sarcoma (REGOSARC): a randomised, double-blind, placebo-controlled, phase 2 
trial. Lancet Oncol 17(12): 1732-1742

21. Duffaud F, Mir O, Boudou-Rouquette P, et al (2019) Efficacy and safety of regorafenib in adult patients with metastatic osteosarcoma: a non-comparative, randomised, double-blind, placebocontrolled, phase 2 study. Lancet Oncol 20(1): 120-133

22. Liu X, Xu J, Li F, et al (2020) Efficacy and safety of the VEGFR2 inhibitor Apatinib for metastatic soft tissue sarcoma: Chinese cohort data from NCT03121846. Biomed Pharmacother 122: 109587

23. Yang S, Zhang Z, Wang Q (2019) Emerging therapies for small cell lung cancer. J Hematol Oncol 12(1): 47

24. Zhou AP, Bai Y, Song Y, et al (2019) Anlotinib Versus Sunitinib as First-Line Treatment for Metastatic Renal Cell Carcinoma: A Randomized Phase II Clinical Trial. Oncologist 24(8): e702-e708

25. Han B, Li K, Zhao Y, et al (2018) Anlotinib as a third-line therapy in patients with refractory advanced non-small-cell lung cancer: a multicentre, randomised phase II trial (ALTER0302). Br J Cancer 118(5): 654-661

26. Sun Y, Du F, Gao M, et al (2018) Anlotinib for the Treatment of Patients with Locally Advanced or Metastatic Medullary Thyroid Cancer. Thyroid 28(11): 1455-1461

27. Chi Y, Fang Z, Hong X, et al (2018) Safety and Efficacy of Anlotinib, a Multikinase Angiogenesis Inhibitor, in Patients with Refractory Metastatic Soft-Tissue Sarcoma. Clin Cancer Res 24(21): 52335238

28. Kampmann E, Altendorf-Hofmann A, Gibis S, et al (2015) VEGFR2 predicts decreased patients survival in soft tissue sarcomas. Pathol Res Pract 211(10): 726-30

29. Xie L, Guo W, Wang Y, Yan T, Ji T, Xu J (2018) Apatinib for advanced sarcoma: results from multiple institutions' off-label use in China. BMC Cancer 18(1): 396

30. Oh CR, Hong JY, Kim JH, et al (2020) Real-World Outcomes of Pazopanib Treatment in Korean Patients with Advanced Soft Tissue Sarcoma: A Multicenter Retrospective Cohort Study. Target Oncol 15(4): 485-493

31. Li T, Wang L, Wang H, et al (2016) A retrospective analysis of 14 consecutive Chinese patients with unresectable or metastatic alveolar soft part sarcoma treated with sunitinib. Invest New Drugs 34(6): 701-706

32. Berry V, Basson L, Bogart E, et al (2017) REGOSARC: Regorafenib versus placebo in doxorubicinrefractory soft-tissue sarcoma-A quality-adjusted time without symptoms of progression or toxicity analysis. Cancer 123(12): 2294-2302

33. Van der Graaf WT, Blay JY, Chawla SP, et al (2012) Pazopanib for metastatic soft-tissue sarcoma (PALETTE): a randomised, double-blind, placebo-controlled phase 3 trial. Lancet 379(9829): $1879-86$

34. Teshima Y, Nomura S, Fukasawa N (2021) Postmarketing observational study of pazopanib in patients with metastatic soft tissue sarcoma in Japan. Jpn J Clin Oncol 51(4): 612-621

35. Karaağaç M, Sezgin Y, Eryılmaz MK, Araz M, Kaplan MA, Artaç M (2020) The real-life outcome of pazopanib in patients with advanced soft tissue sarcoma: A retrospective cross-sectional study of a 
Turkish cohort. J Oncol Pharm Pract 26(7): 1657-1666

36. Zhu B, Li J, Xie Q, Diao L, Gai L, Yang W (2018) Efficacy and safety of apatinib monotherapy in advanced bone and soft tissue sarcoma: An observational study. Cancer Biol Ther 19(3): 198-204

37. Wang Y, Min L, Zhou Y, et al (2019) The efficacy and safety of apatinib in metastatic alveolar soft part sarcoma: a case series of six patients in one institution. Cancer Manag Res 11: 3583-3591

38. George S, Merriam P, Maki RG, et al (2009) Multicenter phase II trial of sunitinib in the treatment of nongastrointestinal stromal tumor sarcomas. J Clin Oncol 27(19): 3154-60

39. Sun Y, Niu W, Du F, et al (2016) Safety, pharmacokinetics, and antitumor properties of anlotinib, an oral multi-target tyrosine kinase inhibitor, in patients with advanced refractory solid tumors. $J$ Hematol Oncol 9(1): 105

40. Lin B, Song X, Yang D, Bai D, Yao Y, Lu N (2018) Anlotinib inhibits angiogenesis via suppressing the activation of VEGFR2, PDGFRß and FGFR1. Gene 654: 77-86

41. Stacchiotti S, Negri T, Zaffaroni N, et al (2011) Sunitinib in advanced alveolar soft part sarcoma: evidence of a direct antitumor effect. Ann Oncol 22(7): 1682-1690

42. Stacchiotti S, Mir O, Le Cesne A, et al (2018) Activity of Pazopanib and Trabectedin in Advanced Alveolar Soft Part Sarcoma. Oncologist 23(1): 62-70

43. Paoluzzi L, Maki RG (2019) Diagnosis, Prognosis, and Treatment of Alveolar Soft-Part Sarcoma: A Review. JAMA Oncol 5(2): 254-260

44. Xie L, Xu J, Sun X, et al (2019) Apatinib for Advanced Osteosarcoma after Failure of Standard Multimodal Therapy: An Open Label Phase II Clinical Trial. Oncologist 24(7): e542-e550

45. In GK, Hu JS, Tseng WW (2017) Treatment of advanced, metastatic soft tissue sarcoma: latest evidence and clinical considerations. Ther Adv Med Oncol 9(8): 533-550

46. Wang X, Liu Z (2018) Systematic meta-analysis of genetic variants associated with osteosarcoma susceptibility. Medicine (Baltimore) 97(38): e12525

47. Bian Z, He Q, Wang X, Li M, Zhu L (2015) Association of genetic polymorphisms with osteosarcoma risk: a meta-analysis. Int J Clin Exp Med 8(6): 8317-28

48. Antonescu CR (2006) The role of genetic testing in soft tissue sarcoma. Histopathology 48(1): 13-21

49. Liu J, Deng YT, Jiang Y (2021) Switch maintenance therapy with anlotinib after chemotherapy in unresectable or metastatic soft tissue sarcoma: a single-center retrospective study. Invest New Drugs 39(2): 330-336

50. Shao L, Wang W, Song Z, Zhang Y (2019) The efficacy and safety of anlotinib treatment for advanced lung cancer. Onco Targets Ther 12: 6549-6554

\section{Tables}

Table 1 Patients' Characteristics 


\begin{tabular}{|lll|}
\hline Characteristics & Cases (n) & Percentage区\%区 \\
\hline gender & & \\
\hline male & 29 & 64.4 \\
\hline female & 16 & 35.6 \\
\hline Disease & & \\
\hline Synovial sarcoma & 8 & 17.8 \\
\hline ASPS & 7 & 15.6 \\
\hline Osteosarcoma & 5 & 11.1 \\
\hline Fibrosarcoma & 5 & 11.1 \\
\hline UPS & 4 & 8.9 \\
\hline Undifferentiated sarcoma & 4 & 8.9 \\
\hline Rhabdomyosarcoma & 2 & 4.4 \\
\hline Leiomyosarcoma & 2 & 4.4 \\
\hline Chordoma & 1 & 2.2 \\
\hline Epithelioid sarcoma & 1 & 2.2 \\
\hline Clear cell sarcoma & 1 & 2.2 \\
\hline Small round cell sarcoma & 1 & 2.2 \\
\hline Ewing sarcoma & 1 & 2.2 \\
\hline Liposarcoma & 1 & 2.2 \\
\hline Tumor location & 25 & 55.6 \\
\hline limbs & 25 & 20.0 \\
\hline trunk & 20 & 44.4 \\
\hline ECOG & & \\
\hline 0 & & \\
\hline 1 & & \\
\hline 2 & & \\
\hline
\end{tabular}

Notes: data are presented as numbers(percentages).

Abbreviations: ASPS, alveolar soft-part sarcoma;UPS, undifferentiated pleomorphic sarcoma; ECOG, Eastern Cooperative Oncology Group Performance Status; 
Table 2 Previous treatment information

\begin{tabular}{|c|c|c|}
\hline & Cases (n) & Percentage (\%) \\
\hline \multicolumn{3}{|c|}{ Sensitive to chemotherapy } \\
\hline Very sensitive & 3 & 6.7 \\
\hline Normal sensitive & 23 & 51.1 \\
\hline Less sensitive & 3 & 6.7 \\
\hline No sensitive & 12 & 26.7 \\
\hline unknown & 3 & 8.9 \\
\hline \multicolumn{3}{|l|}{ Lines of therapy } \\
\hline 1 & 3 & 6.7 \\
\hline 2 & 13 & 38.9 \\
\hline 3 & 17 & 37.8 \\
\hline 4 & 10 & 22.2 \\
\hline 5 & 2 & 4.4 \\
\hline \multicolumn{3}{|c|}{ Treatment methods before Anlotinib } \\
\hline Operation & 36 & 80.0 \\
\hline chemotherapies & 25 & 55.6 \\
\hline radiotherapy & 8 & 17.8 \\
\hline Other TKi drugs & 12 & 26.7 \\
\hline immunotherapy & 2 & 4.4 \\
\hline \multicolumn{3}{|l|}{ Target lesion } \\
\hline Pulmonary nodule & 41 & 93.2 \\
\hline Relapsed tumor & 4 & 6.8 \\
\hline
\end{tabular}

Notes: Data are presented as numbers and percentages.

Table 3. Disease control based on the length of follow-up 


\begin{tabular}{|lll|}
\hline & 3 months & Overall response \\
\hline $\mathrm{PR}(\mathrm{n})$ & 3 & 1 \\
\hline $\mathrm{SD}(\mathrm{n})$ & 33 & 11 \\
\hline $\mathrm{PD}(\mathrm{n})$ & 8 & 32 \\
\hline $\mathrm{ORR}(\%)$ & 6.82 & 2.27 \\
\hline $\mathrm{DCR}(\%)$ & 81.82 & 27.27 \\
\hline
\end{tabular}

Notes: data are presented as numbers or percentages

Abbreviations: PR, Partial response; SD, Stable disease; PD, Progressive disease; ORR, objective response rate; $\mathrm{DCR}$, disease control rate;

Table 4 Adverse events of anlotinib in the treatment of sarcoma 


\begin{tabular}{|c|c|c|}
\hline & Cases $\llbracket n \rrbracket$ & Percentage (\%) \\
\hline hypertension & 2 & 4.4 \\
\hline Grade 1 & 1 & 2.2 \\
\hline Grade 3 & 1 & 2.2 \\
\hline proteinuria & 2 & 4.4 \\
\hline Grade 2 & 1 & 2.2 \\
\hline Grade 3 & 1 & 2.2 \\
\hline anemia & 7 & 15.6 \\
\hline Grade 1 & 6 & 13.3 \\
\hline Grade 2 & 1 & 2.2 \\
\hline leukopenia & 4 & 8.9 \\
\hline Grade 1 & 1 & 2.2 \\
\hline Grade 2 & 2 & 4.4 \\
\hline Grade 4 & 1 & 2.2 \\
\hline thrombocytopenia & 3 & 6.7 \\
\hline Grade 1 & 2 & 4.4 \\
\hline Grade 4 & 1 & 2.2 \\
\hline fatigue & 5 & 11.1 \\
\hline Grade 1 & 3 & 6.7 \\
\hline Grade 2 & 1 & 2.2 \\
\hline Grade 3 & 1 & 2.2 \\
\hline Hand-foot syndrome & 4 & 8.9 \\
\hline Grade 1 & 4 & 8.9 \\
\hline anorexia & 5 & 11.1 \\
\hline Grade 1 & 4 & 8.9 \\
\hline Grade 3 & 1 & 2.2 \\
\hline High TSH & 8 & 17.8 \\
\hline Grade 1 & 5 & 11.1 \\
\hline Grade 2 & 3 & 6.7 \\
\hline
\end{tabular}




\begin{tabular}{|ccc|} 
Dysfunction of liver & 5 & 11.1 \\
\hline Grade 1 & 3 & 6.7 \\
Grade 3 & 2 & 4.4 \\
diarrhea & 3 & 6.7 \\
Grade 1 & 2 & 4.4 \\
Grade 2 & 1 & 2.2 \\
\hline pneumothorax & 3 & 6.7 \\
\hline Grade 2 & 3 & 6.7 \\
\hline
\end{tabular}

Notes: Data are presented as numbers and percentages.

\section{Figures}

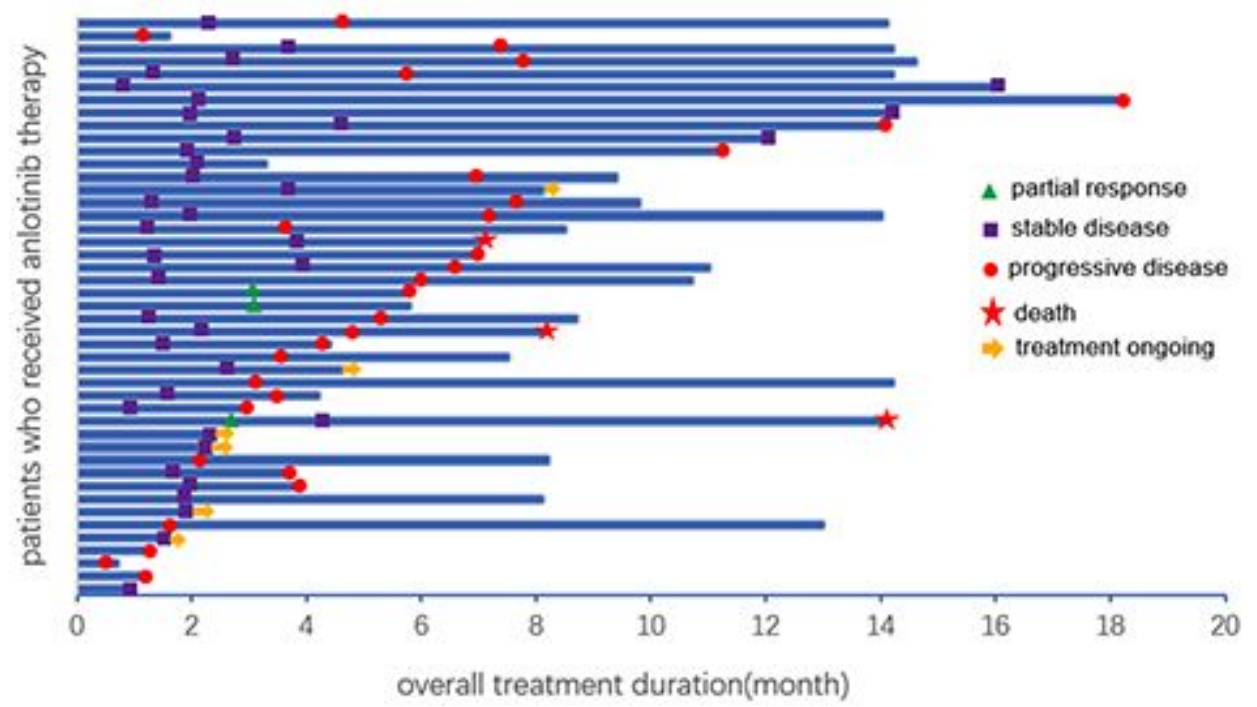

\section{Figure 1}

Overall responses of 45 patients with advanced sarcoma who were treated with anlotinib. Among the 45 patients, one patient were excluded from the efficacy evaluation. The responses were partial response, stable disease, and progressive disease in 1 (2.27\%), 11 (25.00\%), and 32 patients $(72.73 \%)$, respectively 


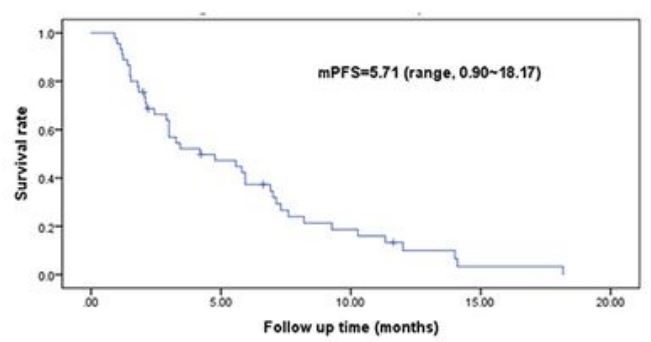

A)

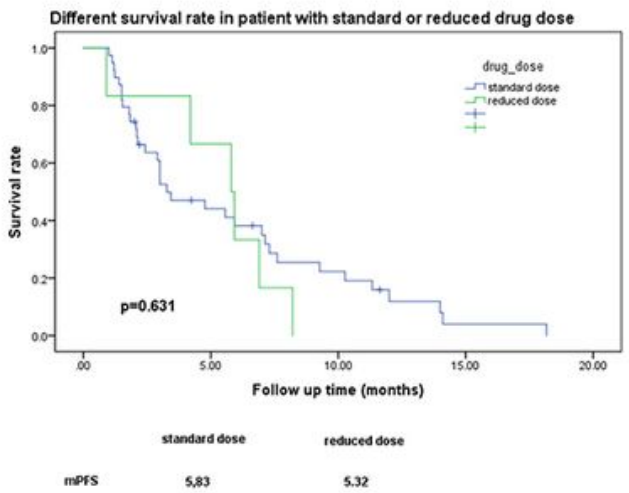

C)

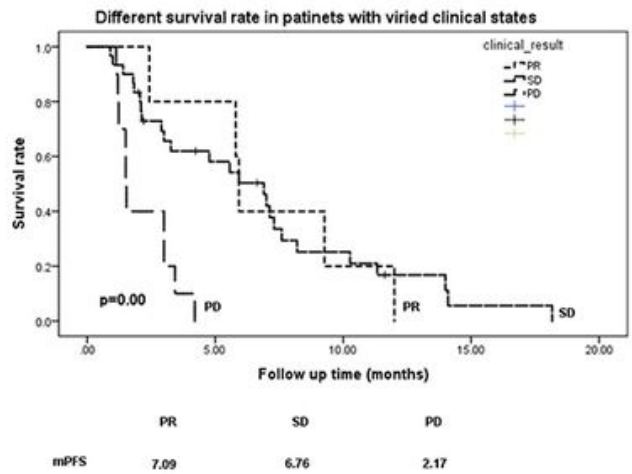

E)

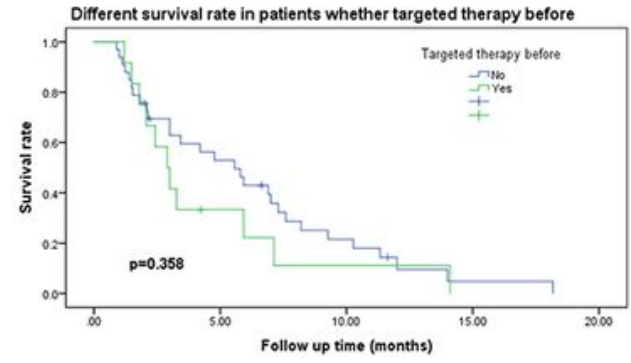

4.53

6.17

B)

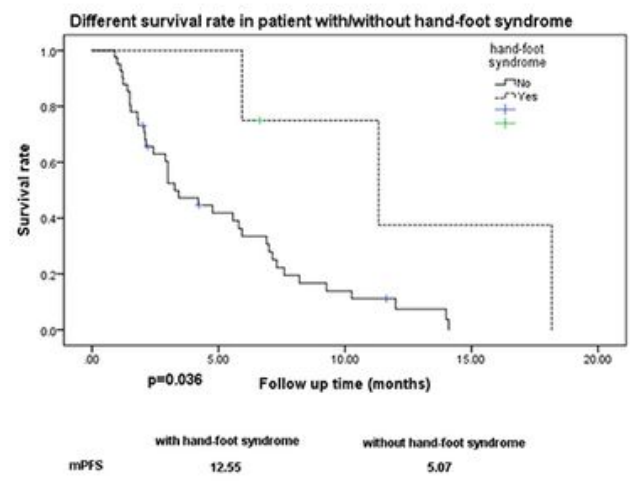

D)

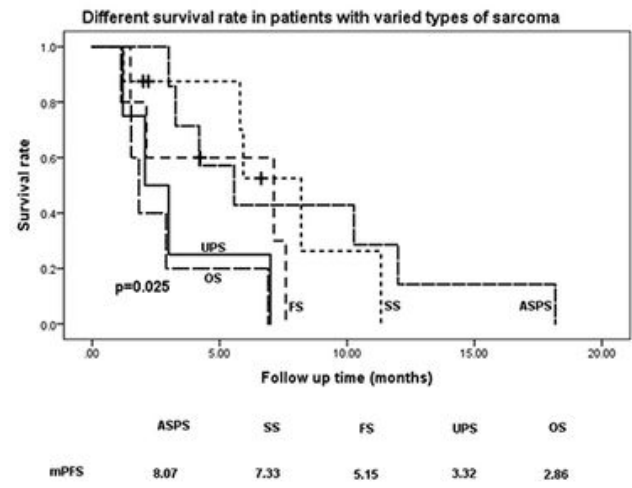

F)

\section{Figure 2}

Efficacy analysis and influencing factors of anlotinib in advanced sarcoma. (a) PFS after anlotinib treatment of advanced sarcoma that did not respond to chemotherapy (mPFS: 5.71 months). (b) There was no statistically significant difference between the history of targeted drug therapy and PFS. (c) There was no statistically significant difference between the dose of anlotinib and PFS. (d) hand-foot syndrome was significantly correlated with longer PFS in this cohort, and patients who suffered from hand-foot 
syndrome during treatment had significantly longer PFS than those without hand-foot syndrome. (e) PFS was significantly prolonged in PR group. (f) There were significant differences among different subtypes

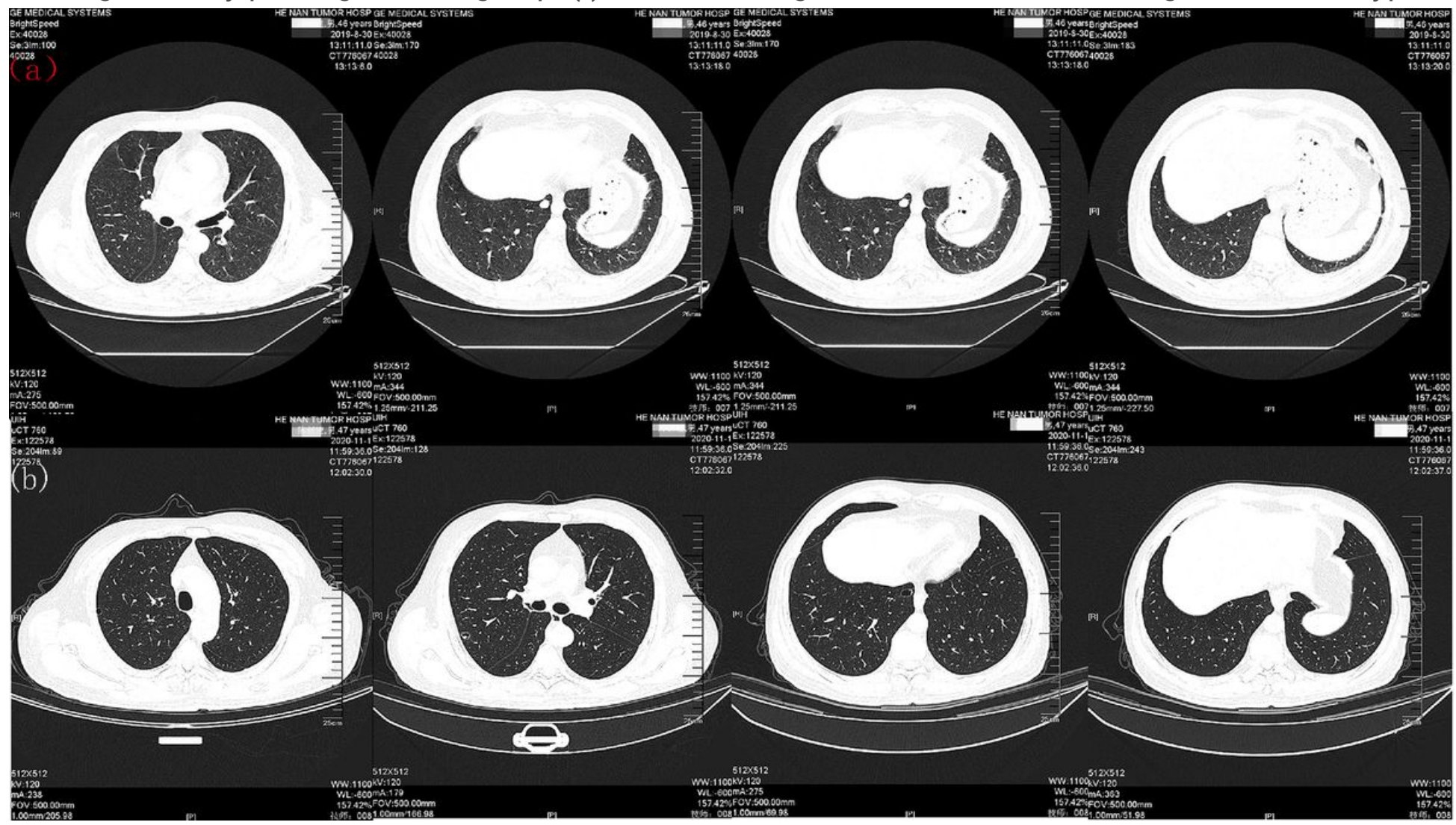

\section{Figure 3}

Series computed tomography scans of a pulmonary metastatic nodule in a 46-year-old male patient. (a)

(b) demonstrate Lung nodes change before and 14 months after anlotinib administration

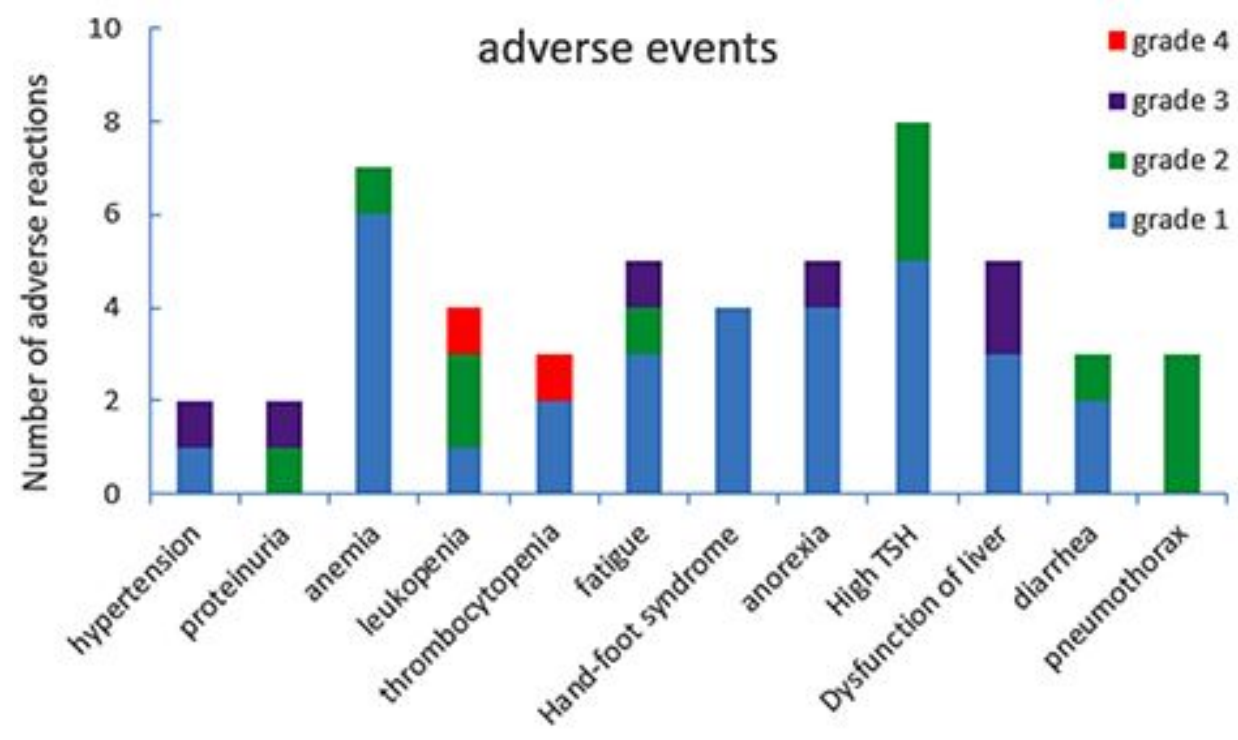

Figure 4

Frequencies and grades of adverse events to anlotinib 